\title{
Accuracy of dopaminergic imaging as a biomarker for mild cognitive impairment with Lewy bodies
}

Gemma Roberts, Paul C. Donaghy, Jim Lloyd, Rory Durcan, George Petrides, Sean J. Colloby, Sarah Lawley, Joanna Ciafone, Calum A. Hamilton, Michael Firbank, Louise Allan, Nicola Barnett, Sally Barker, Kirsty Olsen, Kim Howe, Tamir Ali, John-Paul Taylor, John O'Brien and Alan J. Thomas

\section{Background}

Dopaminergic imaging is an established biomarker for dementia with Lewy bodies, but its diagnostic accuracy at the mild cognitive impairment $(\mathrm{MCl})$ stage remains uncertain.

\section{Aims}

To provide robust prospective evidence of the diagnostic accuracy of dopaminergic imaging at the $\mathrm{MCl}$ stage to either support or refute its inclusion as a biomarker for the diagnosis of $\mathrm{MCl}$ with Lewy bodies.

\section{Method}

We conducted a prospective diagnostic accuracy study of baseline dopaminergic imaging with $\left.\left[{ }^{123}\right]\right] \mathrm{N}-\omega$-fluoropropyl-2$\beta$ -

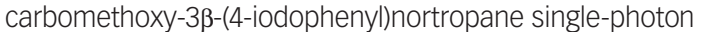
emission computerised tomography $\left({ }^{123}\right.$-FP-CIT SPECT) in 144 patients with $\mathrm{MCl}$. Images were rated as normal or abnormal by a panel of experts with access to striatal binding ratio results. Follow-up consensus diagnosis based on the presence of core features of Lewy body disease was used as the reference standard.

\section{Results}

At latest assessment (mean 2 years) 61 patients had probable $\mathrm{MCl}$ with Lewy bodies, 26 possible $\mathrm{MCl}$ with Lewy bodies and 57 $\mathrm{MCl}$ due to Alzheimer's disease. The sensitivity of baseline FP-CIT visual rating for probable MCI with Lewy bodies was $66 \%$ (95\% Cl 52-77\%), specificity 88\% (76-95\%) and accuracy $76 \%$ (68-84\%), with positive likelihood ratio 5.3 .

\section{Conclusions}

It is over five times as likely for an abnormal scan to be found in probable $\mathrm{MCl}$ with Lewy bodies than $\mathrm{MCl}$ due to Alzheimer's disease. Dopaminergic imaging appears to be useful at the $\mathrm{MCl}$ stage in cases where Lewy body disease is suspected clinically.

\section{Keywords}

Mild cognitive impairment; dopaminergic imaging; diagnostic accuracy; ${ }^{123}$ |-FP-CIT; ioflupane.

\section{Copyright and usage}

(c) The Authors 2020. This is an Open Access article, distributed under the terms of the Creative Commons Attribution licence (http://creativecommons.org/licenses/by/4.0/), which permits unrestricted re-use, distribution, and reproduction in any medium, provided the original work is properly cited.

\section{Background}

Accurate disease stratification is required to enable optimum application of future disease-modifying treatments for dementia. The failure of new treatments in Alzheimer's disease may be related to them being applied too late, and to people without pure Alzheimer's disease pathology, ${ }^{1}$ with Lewy body disease recognised as a common co-pathology even in well-characterised Alzheimer's disease cohorts. ${ }^{2}$ Biomarkers play a crucial role in accurate stratification and dopaminergic imaging is included as an indicative biomarker in the fourth dementia with Lewy bodies (DLB) consensus criteria, alongside cardiac sympathetic innervation imaging and polysomnography. ${ }^{3}$ Although dopaminergic imaging with $\left.{ }^{[123} \mathrm{I}\right] N$ - $\omega$-fluoropropyl-2 $\beta$-carbomethoxy-3 $\beta$-(4-iodophenyl)nortropane single-photon emission computerised tomography $\left({ }^{123} \mathrm{I}\right.$-FP-CIT SPECT) is well-established as a diagnostic marker with good sensitivity and specificity in DLB, the recent Consensus research criteria for prodromal DLB at the mild cognitive impairment (MCI) stage $(\mathrm{MCI}-\mathrm{LB})^{4}$ emphasise the need for prospective studies to assess the diagnostic accuracy of FP-CIT for MCI-LB.

\section{Aims}

Previously we reported our findings in a cohort of 33 patients with probable MCI-LB and 27 with MCI due to Alzheimer's disease (MCI-AD). ${ }^{5}$ We found FP-CIT to have a high specificity of $89 \%$ at the MCI stage (95\% CI 71-98\%), similar to DLB. The sensitivity for detecting probable MCI-LB appeared to be lower than in DLB at $61 \%$ (95\% CI $43-77 \%)$. Here we extend this study by recruiting further patients with MCI in order to improve the precision of our diagnostic accuracy estimates and validate our previous findings. In addition, we carried out cardiac sympathetic innervation imaging on new participants with $\mathrm{MCI}$ and all previous participants with MCI who agreed to return for further scans to provide more certainty for our consensus diagnoses, which are used as reference standard. Our hypothesis was that we would provide more robust prospective evidence that FP-CIT has a high diagnostic accuracy at the MCI stage and thus support its inclusion as a biomarker for MCI-LB diagnosis.

\section{Method}

\section{Study design}

We conducted a single-centre prospective cohort study into the accuracy of ${ }^{123}$ I-FP-CIT SPECT imaging in the diagnosis of probable MCI-LB in patients with one or more clinical symptoms at baseline that could indicate Lewy body disease. All patients were diagnosed with MCI on entry to the study; some developed dementia during follow-up.

Our index test was the dichotomised baseline FP-CIT image consensus panel rating result (see Image acquisition and processing). Our reference standard was consensus clinical diagnosis at most recent assessment of either probable Lewy body disease (comprising probable MCI-LB or probable DLB) or Alzheimer's disease (comprising MCI-AD or Alzheimer's disease dementia). Consensus clinical diagnosis at most recent assessment incorporated core features and cardiac metaiodobenzylguanidine (mIBG) imaging result where available (see Clinical diagnosis). The presence of core clinical features was assessed masked to imaging biomarker results. 
Patients with uncertain diagnoses of possible MCI-LB or possible DLB were included in the study, but not in the main diagnostic accuracy calculation, because of the greater diagnostic uncertainty in this group.

Our primary research question was as follows: what is the sensitivity, specificity and overall accuracy of ${ }^{123}$ I-FP-CIT SPECT for the diagnosis of probable MCI-LB?

\section{Patient recruitment}

Patients aged 60 or older with an existing clinical diagnosis of MCI were recruited from local memory services in the North-East of England between April 2013 and September 2019. The medical records of all patients meeting the above criteria were reviewed to assess eligibility. In addition to the diagnosis of MCI, records had to include one or more clinical symptoms supportive of Lewy body disease (for example mood changes, sleep disturbance or autonomic symptoms) and/or the presence of core DLB features. Written informed consent was obtained from all patients. Following consent, participants underwent interview, clinical assessment and neurological examination by a medical doctor (R.D., S.L.). Determination of parkinsonism for diagnostic purposes was based on the neurological examination.

The MDS Unified Parkinson's Disease Rating Scale - Motor Examination (UPDRS-III), ${ }^{6}$ Epworth Sleepiness $\mathrm{Scale}^{7}$ and Geriatric Depression Scale ${ }^{8}$ were administered to patients. The Instrumental Activities of Daily Living (IADL) scale, ${ }^{9}$ North-East Visual Hallucinations Inventory, ${ }^{10}$ Neuropsychiatric Inventory, ${ }^{11}$ Mayo Sleep Questionnaire, ${ }^{12}$ Clinician Assessment of Fluctuation ${ }^{13}$ and Dementia Cognitive Fluctuation Scale ${ }^{14}$ were administered to spouses or close family members acting as informants. The Clinical Dementia Rating scale $(\mathrm{CDR})^{15}$ was completed using clinical history and research assessments. A detailed neuropsychological evaluation was also carried out as reported in our recent publication ${ }^{16}$ which included the Addenbrooke's Cognitive Examination - Revised, ${ }^{17}$ a 100-point cognitive screening test from which a Mini-Mental State Examination score was derived. Other tests included FAS Verbal Fluency, ${ }^{18}$ the Trail-making Test Parts $\mathrm{A}$ and $\mathrm{B},{ }^{19}$ the Graded Naming Test, ${ }^{20}$ the Rey Auditory Verbal Learning Test ${ }^{21}{ }^{21}$ simple and choice reaction times ${ }^{22}$ and line angle discrimination. ${ }^{16,23}$

Patients recruited from April 2016 onwards were offered cardiac sympathetic innervation imaging with $\mathrm{mIBG}$, the results of which were incorporated into diagnoses. Cardiac mIBG uptake was quantified using the heart-to-mediastinum count ratio as a diagnostic indicator, as described previously. ${ }^{24,25}$

The authors assert that all procedures contributing to this work comply with the ethical standards of the relevant national and institutional committees on human experimentation and with the Helsinki Declaration of 1975, as revised in 2008. All procedures involving human patients were approved by the National Research Ethics Service Committee North East - Newcastle \& North Tyneside 2 (Research Ethics Committee Identification Number 15/NE/0420).

\section{Clinical diagnosis}

A three-person consensus clinical panel of experienced consultant old age psychiatrists (A.J.T., P.C.D., J.-P.T.) independently reviewed the research assessment and clinical notes and confirmed diagnoses of MCI according to National Institute on Aging-Alzheimer's Association (NIA-AA) criteria. $^{26}$ This consensus panel method has previously been validated against autopsy and is recognised by regulatory authorities as the clinical gold standard for living patients. ${ }^{27,28}$ This was based on evidence of minimal functional impairment and a CDR of 0 or 0.5 , and a history of subjective and objective cognitive decline on assessment. Neuropsychological test results were not used to confirm MCI. Anyone with dementia or only subjective impairment was excluded. To determine the aetiology, the presence or absence of core Lewy body features were also rated by the panel, in accordance with the fourth consensus criteria for $\mathrm{DLB}^{3}$ and the recently published consensus research criteria for MCI-LB. ${ }^{4}$ The panel reviewed the notes from the clinical and neurological examination done during the research assessment as well as the health service records for this.

Determination of parkinsonism for diagnostic purposes was based on the presence of bradykinesia (defined as slowness of movement and decrement in amplitude or speed), rest tremor or rigidity. Participants all had baseline research assessments and most had annual review data available by the time of data locking. Annual review data (up to 7 years) was used for the consensus panel diagnosis where available. Cardiac $\mathrm{mIBG}$ results were later incorporated into diagnoses, but the panel decisions on symptom presence were made initially masked to these findings. FP-CIT results were not included in the diagnosis, and the panel had no access to these.

Participants received a diagnosis MCI-AD when they had no core Lewy body features, a normal mIBG scan and evidence of decline that was characteristic of Alzheimer's disease, i.e., they met the additional NIA-AA criterion of 'etiology of MCI consistent with Alzheimer's disease pathophysiologic process'. ${ }^{26}$ Biomarker tests for Alzheimer's pathology were not conducted, in line with research practice when the study was developed.

The study is concerned with the detection of Lewy body disease and the presence of Alzheimer's disease or other aetiologies does not exclude Lewy body disease. Probable MCI-LB was diagnosed in patients with either two or more core Lewy body features, or one or more core feature and abnormal mIBG, in accordance with consensus criteria. ${ }^{4}$ Patients were assigned the diagnosis of possible MCI-LB if they presented with only one core feature and their mIBG scan was normal, or if their mIBG scan result was abnormal but they had no core features.

\section{Exclusion criteria}

Exclusion criteria included the presence of a possible frontotemporal or vascular aetiology, parkinsonism pre-dating onset of cognitive symptoms by over 1 year, history of stroke, major cerebrovascular disease on brain imaging, severe mental illness and either dementia or lack of cognitive impairment at screening. Because we were including cardiac mIBG imaging, we excluded participants taking labetalol and tricyclic antidepressants, if they were not able to safely complete withdrawal $48-72 \mathrm{~h}$ prior to the cardiac mIBG scan, as these are known to affect cardiac mIBG uptake. ${ }^{29}$

We excluded participants with heart failure (New York Heart Association Class II or worse) or myocardial infarction within a year prior to recruitment. Participants were not excluded if they had risk factors for cardiac disease, or less severe heart failure, as these are common features in the older population.

\section{Image acquisition and processing}

Patients were scanned within 1 month of baseline clinical assessment, unless an ${ }^{123}$ I-FP-CIT scan had been acquired for clinical reasons within the previous 6 months, in which case it was not repeated, in accordance with our ethical approvals. This was the case for four patients, whose images were obtained for rating. These clinical images were acquired using a very similar protocol to the study scan protocol below, but not all were acquired on the same gamma camera.

Patients were scanned 3-6 $\mathrm{h}$ following a bolus intravenous injection of $185 \mathrm{MBq}$ of ${ }^{123} \mathrm{I}$-FP-CIT (Ioflupane (DaTSCAN) GE Healthcare, UK) (scan duration, $25 \mathrm{~min}$ ) using a double-headed gamma camera (Siemens Symbia S or Siemens Intevo) fitted with a low-energy high-resolution parallel hole collimator. A total of 120 
(60 per detector) $25 \mathrm{~s}$ views over a $360^{\circ}$ orbit were acquired on a $128 \times$ 128 matrix with a zoom of $1.23 \times$ giving a pixel size $3.9 \mathrm{~mm} \times 3.9 \mathrm{~mm}$. Image processing and display was then performed on a Hermes workstation (Hermes Medical Solutions, Stockholm, Sweden).

Images used in our previous publication were reconstructed without attenuation correction using filtered back projection and a Butterworth filter (order 10, cut-off 1.3 cycles/cm). New FP-CIT images were reconstructed using iterative reconstruction with resolution recovery, uniform attenuation correction and Monte Carlo scatter correction. For all images, transverse data was manually re-oriented to correct for any head tilt and to provide a consistent display.

\section{Visual rating of FP-CIT images}

Visual assessment of all scans was undertaken masked to clinical diagnosis and information. Briefly, scans were rated independently by each panel member using an established FP-CIT visual rating procedure $^{30}$ that has also shown diagnostic value in the differential diagnosis of DLB and Alzheimer's disease. ${ }^{31}$ Raters were provided with age-corrected specific binding ratio results generated using DaTQUANT v1.0 (GE Healthcare, Chalfont St Giles, UK) prior to April 2016 and BRASS v2.5 (Hermes Medical Ltd, Stockholm, Sweden) for more recent scans. The consensus panel consisted of a group of four or five raters experienced at reviewing FP-CIT images: A.J.T., J.L., P.C.D. and G.P.; G.R. from 2016. The panel members were sent sets of anonymised images to review in a randomised order by an independent member of the team (S.J.C.). Panel members used their professional judgement in cases where visual assessment and semi-quantification did not agree.

Each rater independently dichotomised the scans as normal (non-Lewy body appearance) or abnormal. Mild balanced loss of dopaminergic uptake throughout both striata was designated as within normal limits, as this pattern was seen in controls in our paper using autopsy-confirmed diagnoses. ${ }^{32}$ Moderate-to-severe balanced loss was rated abnormal. After rating all scans, any scan where there was not agreement between at least four raters was then subsequently reviewed at a panel meeting, where a full consensus rating of normal or abnormal was agreed. If an infarct along the nigro-striatal pathway was suspected to be affecting uptake, images from magnetic resonance imaging (MRI) were reviewed retrospectively and the participant excluded if confirmed. Example images categorised as normal and abnormal are shown in Supplementary Figure 1 available at https://doi.org/10.1192/bjp.2020.234.

\section{Statistical analysis}

The Statistical Package for Social Sciences software (SPSS version 25) was used to produce summary statistics. Continuous variables were analysed for differences between the MCI-AD and probable

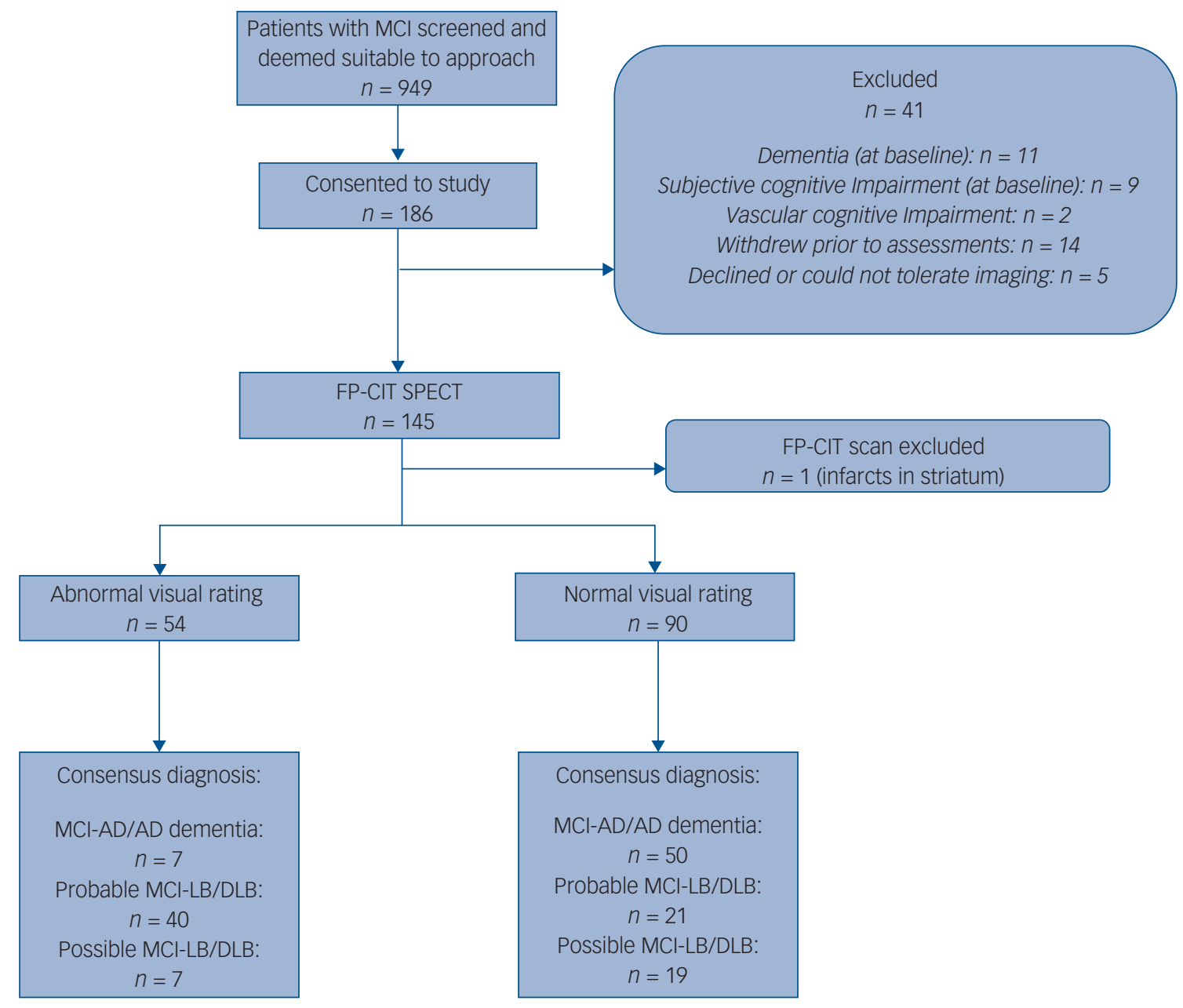

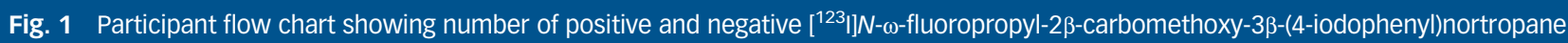
${ }^{123}$ I-FP-CIT) scans and reference standard consensus clinical diagnoses.

AD, Alzheimer's disease; DLB, dementia with Lewy bodies; MCI-AD, mild cognitive impairment (MCI) due to Alzheimer's disease; MCI-LB, MCI with Lewy bodies; SPECT, singlephoton emission computerised tomography. 


\begin{tabular}{|c|c|c|c|}
\hline & $\mathrm{MCl}-\mathrm{AD}$ & Probable $\mathrm{MCl}-\mathrm{LB}$ & $P$ \\
\hline$n$ & 57 & 61 & \\
\hline Women, $n(\%)$ & $32(56)$ & $13(21)$ & $<0.01$ \\
\hline Age at consent, mean (s.d.) & $76.9(7.3)$ & $74.6(7.1)$ & 0.09 \\
\hline Years in study, mean (range) & $1.2(0$ to 5$)$ & $2.0(0$ to 7$)$ & 0.01 \\
\hline Unified Parkinson's Disease Rating Scale - total, mean (s.d.) & $16.1(12.2)$ & $22.0(14.9)$ & 0.03 \\
\hline Mini-Mental State Examination, mean (s.d.) & $26.5(2.4)$ & $26.5(2.1)$ & 0.99 \\
\hline Addenbrooke's Cognitive Examination - Revised total, mean (s.d.) & $79.9(10.2)$ & $81.1(9.0)$ & 0.50 \\
\hline Epworth Sleepiness Scale, mean (s.d.) & $5.2(3.9)$ & $9.4(5.0)$ & $<0.01$ \\
\hline Geriatric Depression Scale, mean (s.d.) & $3.1(2.5)$ & $4.5(3.9)$ & 0.06 \\
\hline Instrumental Activities of Daily Living, mean (s.d.) & $7.1(1.3)$ & $6.3(1.6)$ & 0.01 \\
\hline Clinical Dementia Rating scale, mean (s.d.) & $0.5(0.1)$ & $0.4(0.2)$ & 0.05 \\
\hline Neuropsychiatric Inventory, mean (s.d.) & $7.2(8.2)$ & $14.8(11.8)$ & $<0.01$ \\
\hline Memantine, $n(\%)$ & $1(2)$ & $1(2)$ & 0.96 \\
\hline Cholinesterase inhibitor, $n$ (\%) & $13(23)$ & $31(51)$ & $<0.01$ \\
\hline Anti-Parkinsonian drug, $n$ (\%) & 0 & $9(15)$ & $<0.01$ \\
\hline Fluctuations (baseline), $n$ (\%) & 0 (by definition) & $30(49)$ & $<0.01$ \\
\hline Visual hallucinations (baseline), $n$ (\%) & 0 (by definition) & $16(26)$ & $<0.01$ \\
\hline Parkinsonism (baseline), $n$ (\%) & 0 (by definition) & $23(38)$ & $<0.01$ \\
\hline REM sleep behaviour disorder (baseline), $n$ (\%) & 0 (by definition) & $41(67)$ & $<0.01$ \\
\hline
\end{tabular}

MCI-LB groups using Student's $t$-test or Mann-Whitney $U$-test for independent samples. The $\chi^{2-}$ test was used for determining whether there was a difference in the proportions of binary variables.

BRASS quantification was used to calculate FP-CIT whole striatum and putaminal specific binding ratios (SBRs) for all participants. We checked for difference in mean SBR between the probable MCI-LB and MCI-AD population using an independent samples $t$-test, as data was normally distributed. We tested for a difference in the proportion of abnormal scans in the probable MCI-LB group and the MCI-AD group using a $\chi^{2}$-test. The accuracy of semiquantification alone was calculated from the proportion of scans in each diagnostic group with $Z$-scores below -2, i.e. more than 2 s.d. below the mean of age-matched controls in the BRASS database.

The diagnostic accuracy of FP-CIT visual rating as a biomarker for probable MCI-LB (sensitivity, specificity and overall accuracy values) was calculated from a $2 \times 2$ frequency table. Likelihood ratios were calculated to estimate the added value of dopaminergic imaging in the diagnosis of probable MCI-LB. As a secondary analysis we assessed whether sensitivity appeared greater in those patients with parkinsonism at baseline, compared with those without, recognising that the study would not necessarily be powered to detect a significant difference.

To assess the potential impact of a positive FP-CIT result on diagnosis in clinical practice, we reviewed our probable MCI-LB group, identifying those with fewer than two core features at baseline. From this subset we calculated the proportion with a positive FP-CIT scan.

\section{Results}

A total of 186 patients with MCI consented to take part and were eligible after initial screening; 41 patients later withdrew or were excluded, or the FP-CIT was not done (see flow chart in Fig. 1). One FP-CIT scan was excluded during visual rating because of infarcts in the basal ganglia, confirmed on review of the MRI. Our final group of 144 patients with MCI consisted of 61 participants with probable MCI-LB (or DLB if progressed to dementia during follow-up), 26 with possible MCI-LB or DLB, and 57 with MCI$\mathrm{AD}$ or Alzheimer's disease dementia. In total, 94 of the patients underwent cardiac ${ }^{123} \mathrm{I}$-mIBG scanning. No adverse effects from the FP-CIT or mIBG scans were reported. The demographic and clinical characteristics of the patient groups are given in Table 1.
As seen in clinical practice, the probable MCI-LB group had a higher proportion of men $(P<0.01)$. The sensitivity for detecting probable MCI-LB was $66 \%$ (95\% CI 52-77\%), specificity $88 \%$ (76$95 \%$ ) and accuracy $76 \%$ (68-84\%) (Table 2). The positive likelihood ratio was 5.3 and negative likelihood ratio 0.39 . The percentages of abnormal scans in the probable MCI-LB and MCI-AD groups were $66 \%$ and $12 \%$, respectively, this difference in proportions of abnormal FP-CIT scans was statistically significant $(P<0.001)$.

Examining the 23 participants with probable MCI-LB with parkinsonism and 38 without parkinsonism at the time of the scan, showed a higher proportion of abnormal FP-CIT scans in the group with parkinsonism: $83 \%$ v. 55\%. Fisher's exact test shows this is of borderline significance $(P=0.05)$.

The mean whole striatum SBRs were as follows: MCI-AD: 2.77 (s.d. =0.46); probable MCI-LB: 2.21 (s.d. = 0.66); possible MCI-LB: 2.71 (s.d. =0.55). Three individuals with probable MCI-LB and one with possible MCI-LB were excluded from the SBR analyses as their FP-CIT data was obtained on a different gamma camera shortly before recruitment, as part of routine clinical care. These patients were included in the main visual rating analysis.

The difference in the mean SBR between the MCI-AD group and probable MCI-LB group was 0.56 , which is statistically significant $(P<0.001$, equal variances not assumed). Dot plots of the SBR results for MCI-AD, possible MCI-LB and probable MCI-LB are shown in Fig. 2. The sensitivity of semi-quantification alone for detecting probable MCI-LB was $43 \%(30-57 \%)$, specificity $93 \%$ (83-98\%) and accuracy 68\% (58-76\%) for the lowest striatum $Z$-score. For the lowest putamen the sensitivity was 47\% (33$60 \%)$, specificity $96 \%(88-100 \%)$ and accuracy $71 \%(62-79 \%)$.

Table 2 Contingency table showing numbers of normal and abnormal $\left.\left[{ }^{123}\right]\right] N$ - $\omega$-fluoropropyl-2 $\beta$-carbomethoxy-3 $\beta$-(4-iodophenyl)nortropane scans in the mild cognitive impairment due to Alzheimer's disease (MCl-AD) and probable mild cognitive impairment with Lewy bodies (MCl-LB) and possible MCl-LB groups

\begin{tabular}{|lccc|}
\hline & $\begin{array}{c}\text { Normal, } \\
n(\%)\end{array}$ & $\begin{array}{c}\text { Abnormal, } \\
n(\%)\end{array}$ & Totals, $n$ \\
\hline $\begin{array}{l}\text { MCl-AD/Alzheimer's disease } \\
\text { Probable MCl-LB/dementia } \\
\quad \text { with Lewy bodies }\end{array}$ & $50(88)$ & $7(12)$ & 57 \\
$\begin{array}{c}\text { Possible MCl-LB/dementia with } \\
\text { Lewy bodies }\end{array}$ & $21(34)$ & $40(66)$ & 61 \\
Totals & $19(73)$ & $7(27)$ & 26 \\
\hline
\end{tabular}


(a) $\square$ MCl-AD $\quad \diamond$ Possible MCl-LB

$\triangle$ Probable MCL-LB

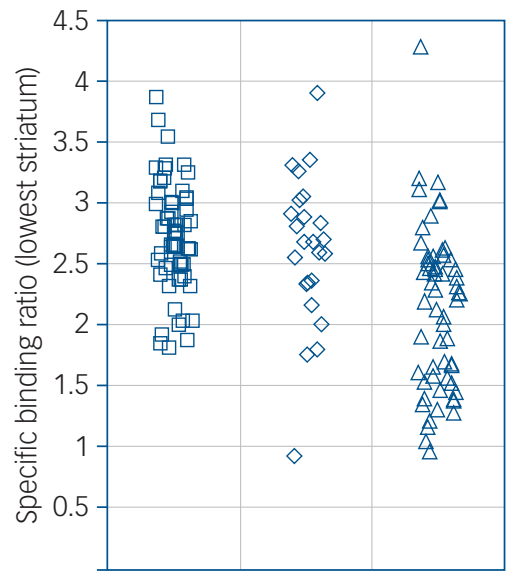

(b) $\square \mathrm{MCl}-\mathrm{AD} \quad \diamond$ Possible MCl-LB

$\triangle$ Probable MCL-LB

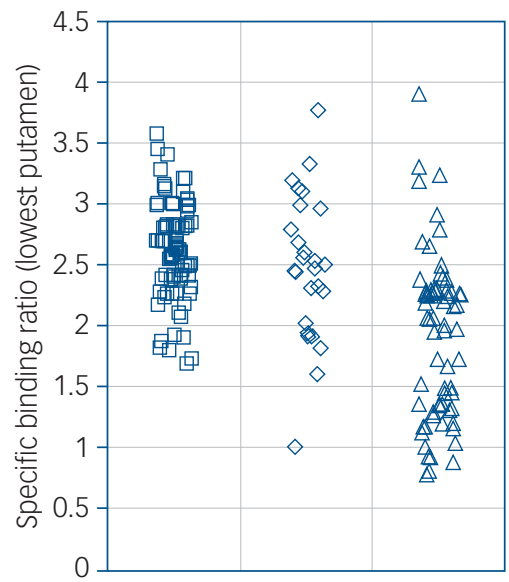

Fig. 2 Plots of specific binding ratio for the mild cognitive impairment (MCI) due to Alzheimer's disease (MCl-AD) and probable $\mathrm{MCl}$ with Lewy bodies (MCl-LB) groups.

(a) Shows the lowest whole striatum specific binding ratio (SBR) and (b) the SBR for the lowest putaminal subregion.

Of the 61 participants with follow-up diagnoses of probable MCI-LB, 25 (41\%) had less than two core features present at baseline. Of these, $15(60 \%)$ had an abnormal baseline FP-CIT scan result.

\section{Discussion}

In this study we report the diagnostic accuracy of ${ }^{123}$ I-FP-CIT in a large group of 144 patients with MCI, including 61 patients with probable MCI-LD and 57 patients with MCI-AD. The strengths of our study include the prospective design and relatively large MCI groups with thorough consensus clinical assessment. A further strength is that we were able to add cardiac mIBG to our protocol for a proportion of participants, which as an established biomarker enhanced the overall quality of our diagnostic assessments.

The sensitivity of FP-CIT consensus visual rating for detecting probable MCI-LB was $66 \%$ (95\% CI $52-77 \%$ ), specificity $88 \%$ (76-95\%) and overall accuracy $76 \%(68-84 \%)$. The positive likelihood ratio of 5.3 means it is five times more likely for an abnormal scan to be found in probable MCI-LB than MCI-AD, showing the test to be useful at the MCI stage where Lewy body disease is suspected clinically. Use of dopaminergic imaging would help identify people with Lewy body disease in MCI cohorts, thereby improving disease-specific stratification and enabling disease-modifying therapies to focus on the relevant target disease. Early identification could also allow for earlier symptomatic intervention and planning, keeping those patients with MCI who are at high risk of converting to DLB under medical review.

Although the specificity of $88 \%$ is high, the relatively low prior probability of a patient having MCI-LB outside a specialist setting means that in practice FP-CIT is only suitable for patients where there is good reason to suspect they may have Lewy body disease. It would, for example, not be appropriate to screen a general group of patients with MCI for MCI-LB with FP-CIT as many false positives would arise, even with the high specificity.

Our secondary analysis suggested that a positive FP-CIT scan is more likely in patients with probable MCI-LB with parkinsonism among the core features, compared with those without parkinsonism at baseline. However, this finding was of borderline significance
$(P=0.05)$ and should be interpreted with caution. It is of note that over half of those without parkinsonism still had abnormal FP-CIT scans, suggesting that dopaminergic deficit can precede overt clinical parkinsonism in MCI. A recent retrospective study of 13 patients with MCI that progressed to Parkinson's disease or DLB showed that all had baseline dopaminergic deficits. ${ }^{33}$

Our further subanalysis assessed the added value of a positive FP-CIT scan in people with probable MCI-LB at latest assessment but less than two core features present at baseline. We found that $60 \%$ of this subgroup (15/25 patients) had a positive FP-CIT result, suggesting FP-CIT may be of benefit in less certain cases where biomarkers are most required. In most clinical situations, patients would not be reviewed by multiple Lewy body disease specialists, so it may be that fewer core features would be identified in clinical practice at baseline, increasing the added value of dopaminergic imaging.

Despite comparable cognitive function, individuals in the probable MCI-LB group were more likely to be in receipt of cholinesterase inhibitors at baseline, consistent with recommendations and their local use in treating neuropsychiatric symptoms of Lewy body disease. ${ }^{34}$ The IADL score was slightly lower in the MCI-LB group than in MCI-AD one, despite similar cognition. This is expected as the extra physical impairment in those with parkinsonism is likely to lower the scores.

We showed significantly lower DaT binding in the probable MCI-LB group than in the MCI-AD group $(P<0.001)$, despite substantial overlap between the groups. Similar results were shown by Kasanuki et al, ${ }^{35}$ who studied a rather different cohort of patients with MCI who had parkinsonism but without cognitive fluctuations or hallucinations. They did not dichotomise the scans into normal and abnormal so accuracy cannot be compared. Compared to our consensus visual rating method, the accuracy of semi-quantification alone was similar, with lower sensitivity and higher specificity. Semi-quantification could therefore be useful in conservatively selecting patients with Lewy body disease for clinical trials, where high specificity is key. However, our visual rating method with the aid of semi-quantification is more reflective of clinical practice, as a scan report is never based on semi-quantification results alone. We cannot compare the accuracy of visual rating alone with semiquantification as we had access to the semi-quantification results when rating the scans. Longer follow-up of the study participants 
whose consensus rating was abnormal and quantification normal will help to clarify if these were abnormal scans.

The distribution of the SBRs of the probable MCI-LB and MCIAD groups shows a significant overlap between these groups, which suggests that many of patients with probable MCI-LB either do not have Lewy body disease affecting the substantia nigra, or this is not sufficient at this early stage to affect dopaminergic function. Some participants may be misdiagnosed and not have Lewy body disease at all; however, the longitudinal follow-up helps to strengthen diagnostic certainty. We feel it is more likely that Lewy body disease in the majority of these cases is manifest outside the nigro-striatal pathway. It is common for patients with DLB to be diagnosed without parkinsonism and it has been reported previously that even by death, $10 \%$ of autopsy-confirmed DLB cases had no nigral involvement. ${ }^{36}$

\section{Limitations}

Limitations of our study include the use of consensus clinical diagnosis as gold standard, rather than histopathology following death. However, thus far five participants with MCI have died and had autopsy assessments. Two with probable MCI-LB both had neocortical Lewy body disease and three with MCI-AD all met standard criteria for Alzheimer's disease (including all Braak stages five and six). This provides some early validation for our diagnoses. Also, the specificity may be higher as our MCI-AD may have Lewy body disease which is not yet manifest in any core features or on cardiac mIBG imaging. Other studies have demonstrated that it is common for patients with a clinical diagnosis of Alzheimer's disease to have Lewy body pathology post-mortem. ${ }^{2}$ We did not use specific Alzheimer's disease biomarkers in this study, as the focus of the study was the identification of Lewy body disease we did not seek to exclude people with concomitant Alzheimer's disease from the MCI-LB group.

Although our findings provide evidence that FP-CIT imaging is diagnostically useful at the MCI stage, they only apply to patients where one or more core or supportive clinical Lewy body features are present and we do not encourage the use of FP-CIT more widely in memory services.

It is postulated that imaging biomarkers of Lewy body disease correlate better with eventual pathology at autopsy than clinical diagnoses, with a 2015 study showing less than $10 \%$ discrepant cases between dopamine PET and pathological findings. ${ }^{37}$ In a previous study we also found that FP-CIT was more accurate than clinical diagnosis. ${ }^{36}$ We attempted to mitigate for this by incorporating mIBG findings, where available, as well as an expert panel approach to increase diagnostic certainty. The exclusion of patients with possible MCI-LB with unclear underlying pathology also increased diagnostic certainty.

In summary, the results of this single-centre study support the 2020 consensus recommendations on the diagnosis of MCI-LB, ${ }^{4}$ providing evidence that dopaminergic imaging is useful in clinical practice even at the MCI stage, with an abnormal scan highly suggestive of MCI-LB.

Gemma Roberts (D), PhD, Translational and Clinical Research Institute

Newcastle University, UK; and Nuclear Medicine Department, Royal Victoria Infirmary, UK; Paul C. Donaghy, PhD, Translational and Clinical Research Institute, Newcastle University, UK; Jim Lloyd, PhD, Translational and Clinical Research Institute, Newcastle University, UK; and Nuclear Medicine Department, Royal Victoria Infirmary, UK;

Rory Durcan, MRCPI, MSC, Translational and Clinical Research Institute, Newcastle

University, UK; George Petrides, FRCR, Nuclear Medicine Department, Royal Victoria University, UK; George Petrides, FRCR, Nuclear Medicine Department, Royal Victoria
Infirmary, UK; Sean J. Colloby, PhD, Translational and Clinical Research Institute, Newcastle University, UK; Sarah Lawley, MBBS, Translational and Clinical Research Institute, Newcastle University, UK; Joanna Ciafone, PhD, Translational and Clinical Research Institute, Newcastle University, UK; Calum A. Hamilton, MSC, Translational and Clinical Research Institute, Newcastle University, UK; Michael Firbank, PhD, Translational and Clinical Research Institute, Newcastle University, UK; Louise Allan, PhD, University of Exeter Medical School, University of Exeter, UK; Nicola Barnett, MSc,
Translational and Clinical Research Institute, Newcastle University, UK; Sally Barker, BSC, Translational and Clinical Research Institute, Newcastle University, UK; Kirsty OIsen, BSC, Translational and Clinical Research Institute, Newcastle University, UK; Kim Howe, BSc, Nuclear Medicine Department, Royal Victoria Infirmary, UK; Tamir Ali, FRCR, Nuclear Medicine Department, Royal Victoria Infirmary, UK; John-Paul Taylor, PhD, Translational and Clinical Research Institute, Newcastle University, UK; John O'Brien, DM, Department of Psychiatry, University of Cambridge school of Clinical Medicine, UK; Alan J. Thomas, PhD, Translational and Clinical Research Institute, Newcastle University, UK

Correspondence: Gemma Roberts. Email: Gemma.roberts@newcastle.ac.uk

First received 17 Aug 2020, final revision 26 Oct 2020, accepted 27 Oct 2020

\section{Supplementary material}

To view supplementary material for this article, please visit http://dx.doi.org/10.1192/bjp.2020.234.

\section{Data availability}

The data that support the findings of this study are available from the corresponding author, G.R., upon reasonable request.

\section{Acknowledgements}

The authors would like to thank the people living with $\mathrm{MCl}$ and the family members who participated in this study for giving up so much of their time. We thank Miss Helen Kain, research support secretary for her ongoing support with patient liaison and data entry for the study, the Nuclear Medicine department at the Newcastle upon Tyne Hospitals NHS Foundation Trust for scanning the participants and welcoming our research staff, and the staff of the National Institute for Health Research (NIHR) Clinical Research Network North East and Cumbria for their invaluable support with participant recruitment for this study.

\section{Author contributions}

G.R.: research project: execution; statistical analysis: design; statistical analysis: execution; manuscript: writing of the first draft. P.C.D.: research project: organisation; research project: execution; statistical analysis: review and critique; manuscript: review and critique. J.L.: research project: conception; research project: execution; statistical analysis: design; statistical analysis: review and critique; manuscript: review and critique. R.D.: research project: execution; statistical analysis: execution; manuscript: review and critique. G.P.: research project: conception; research project: execution; manuscript: review and critique.

S.J.C.: research project: organisation; research project: execution; statistical analysis: design; manuscript: review and critique. S.L.: research project: organisation; research project: execution; manuscript: review and critique. J.C.: research project: organisation; research project: execution; manuscript: review and critique. C.A.H.: research project: organisation; research project: execution; manuscript: review and critique. M.F.: research project: conception; statistical analysis: design; statistical analysis: review and critique; manuscript: review and critique. L.A.: research project: conception; statistical analysis: design; manuscript: review and critique. N.B.: research project: organisation; research project: execution; manuscript: review and critique. S.B.: research project: organisation; research project: execution; manuscript: review and critique. K.O.: research project: organisation; research project: execution; manuscript: review and critique. K.H.: research project: organisation; research project: execution; manuscript: review and critique. T.A.: research project: organisation; research project: execution; manuscript: review and critique. J.-P.T.: research project: conception; research project: execution; statistical analysis: design; statistical analysis: review and critique; manuscript: review and critique. J.O'B. research project: conception; research project: execution; statistical analysis: design; statistical analysis: review and critique; manuscript: review and critique. A.J.T.: research project: conception; research project: execution; statistical analysis: design; statistical analysis: review and critique; manuscript: writing of the first draft; manuscript: review and critique.

\section{Funding}

This study was funded by a major project research grant from Alzheimer's Research UK (ARUKPG2015-13) and by the National Institute for Health Research (NIHR) Newcastle Biomedical Research Centre. We acknowledge support for this investigator-led study from GE Healthcare who provided ligand for the FP-CIT scans. G.R. was supported by an Alzheimer's Society healthcare professional fellowship (2016-2019). Infrastructure and support is provided to authors based at Newcastle by the NIHR Newcastle Biomedical Research Centre, a partnership between Newcastle upon Tyne Hospitals NHS Foundation Trust and Newcastle University. J.O'B. is supported by the NIHR Cambridge Biomedical Research Centre and the Cambridge Centre for Parkinson's Plus.

\section{Declaration of interest}

G.R. and G.P. have received honoraria from GE Healthcare for delivering educational workshops on FP-CIT imaging. J.-P.T. has received honoraria from GE Healthcare for delivering educational presentations on Lewy body disease and has consulted for Sosei-Heptares and Kyowa-Kirin. J.O'B. has acted as a consultant for Axon Neuroscience, TauRX, GE Healthcare, Lilly and Eisai, has been a recipient of grant support from Alliance Medical, GE Healthcare and Merck and received honoraria for talks for GE Healthcare. A.J.T. has received support for 
investigator-led studies and honoraria from GE Healthcare. All other authors declare no conflicts of interest.

\section{References}

1 Jack Jr CR, Bennett DA, Blennow K, Carrillo MC, Dunn B, Haeberlein SB, et al. NIA-AA Research Framework: toward a biological definition of Alzheimer's disease. Alzheimers Dement 2018; 14: 535-62.

2 Toledo JB, Cairns NJ, Da X, Chen K, Carter D, Fleisher A, et al. Clinical and multimodal biomarker correlates of ADNI neuropathological findings. Acta Neuropathol Commun 2013; 1: 65.

3 McKeith IG, Boeve BF, Dickson DW, Halliday G, Taylor JP, Weintraub D, et al. Diagnosis and management of dementia with Lewy bodies: fourth consensus report of the DLB Consortium. Neurology 2017; 89: 88-100.

4 McKeith I, Ferman T, Thomas A, Blanc F, Boeve B, Fujishiro H, et al. Research Criteria for the diagnosis of prodromal dementia with Lewy bodies. Neurology 2020; 94: 743-55.

5 Thomas AJ, Donaghy P, Roberts G, Colloby SJ, Barnett NA, Petrides GS, et al. Diagnostic accuracy of dopaminergic imaging in prodromal dementia with Lewy bodies. Psychol Med 2019; 49: 396-402.

6 Goetz CG, Tilley BC, Shaftman SR, Stebbins GT, Fahn S, Martinez-Martin P, et al Movement Disorder Society-sponsored revision of the Unified Parkinson's Disease Rating Scale (MDS-UPDRS): scale presentation and clinimetric testing results. Mov Disord 2008; 23: 2129-70.

7 Johns MW. A new method for measuring daytime sleepiness: the Epworth sleepiness scale. Sleep 1991; 14(6): 540-5.

8 D'Ath P, Katona P, Mullan E, Evans S, Katona C. Screening, detection and management of depression in elderly primary care attenders. I: The acceptability and performance of the 15 item Geriatric Depression Scale (GDS15) and the development of short versions. Fam Pract 1994; 11: 260-6.

9 Lawton MP, Brody EM. Assessment of older people: self-maintaining and instrumental activities of daily living. Gerontologist 1969; 9: 179-86.

10 Holiday KA, Pirogovsky-Turk E, Malcarne VL, Filoteo JV, Litvan I, Lessig SL, et al. Psychometric properties and characteristics of the north-east visual hallucinations interview in Parkinson's disease. Mov Disord Clin Pract 2017; 4(5): 71723.

11 Cummings JL, Mega M, Gray K, Rosenberg-Thompson S, Carusi DA, Gornbein J. The neuropsychiatric inventory: comprehensive assessment of psychopathology in dementia. Neurology 1994; 44: 2308-14.

12 Boeve BF, Molano JR, Ferman TJ, Smith GE, Lin S-C, Bieniek K, et al. Validation of the Mayo Sleep Questionnaire to screen for REM sleep behavior disorder in an aging and dementia cohort. Sleep Med 2011; 12: 445-53.

13 Walker MP, Ayre GA, Cummings JL, Wesnes K, Mckeith I, O'brien JT, et al. The clinician assessment of fluctuation and the one day fluctuation assessment scale. Two methods to assess fluctuating confusion in dementia. $\mathrm{Br} \mathrm{J}$ Psychiatry 2000; 177: 252-56.

14 Lee DR, Mckeith I, Mosimann U, Ghosh-Nodial A, Grayson L, Wilson B, et al. The dementia cognitive fluctuation scale, a new psychometric test for clinicians to identify cognitive fluctuations in people with dementia. Am J Geriatr Psychiatry 2014; 22: 926-35.

15 Hughes CP, Berg L, Danziger WL, Coben LA, Martin RL. A new clinical scale for the staging of dementia. Br J Psychiatry 1982; 140: 566-72.

16 Donaghy PC, Ciafone J, Durcan R, Hamilton CA, Barker S, Lloyd JJ, et al. Mild cognitive impairment with Lewy bodies: neuropsychiatric supportive symptoms and cognitive profile. Psychol Med [Epub ahead of print] 25 Aug 2020. Available from: https://doi.org/10.1017/S0033291720002901.

17 Mioshi E, Dawson K, Mitchell J, Arnold R, Hodges JR. The Addenbrooke's Cognitive Examination Revised (ACE-R): a brief cognitive test battery for dementia screening. Int J Geriatr Psychiatry 2006; 21: 1078-85.

18 Borkowski JG, Benton AL, Spreen O. Word fluency and brain damage. Neuropsychologia 1966; 5: 135-40.
19 Reitan RM. The relation of the trail making test to organic brain damage. J Consult Psychol 1955; 19: 393-94.

20 McKenna P, Warrington EK. Graded Naming Test: Object Picture Book. Cambridge Cognition Ltd, 2007.

21 Rey A. L'examen clinique en psychologie. Presses Universitaires de France, 1964.

22 Ballard C, O'Brien J, Gray A, Cormack F, Ayre G, Rowan E, et al. Attention and fluctuating attention in patients with dementia with Lewy bodies and Alzheimer disease. Arch Neurol 2001; 58: 977-82.

23 Firbank MJ, Mosimann UP, Watson R, Barber R, Blamire AM, et al. Testing visual perception in dementia with Lewy bodies and Alzheimer disease. Am J Geriatr Psychiatry 2013; 21: 501-8.

24 Roberts G, Kane JPM, Lloyd JJ, Petrides GS, Howe K, O'Brien JT, et al. A comparison of visual and semiquantitative analysis methods for planar cardiac $^{123}$ MIBG scintigraphy in dementia with Lewy bodies. Nucl Med Commun 2019; 40: 734-43.

25 Roberts G, Lloyd JJ, Kane JPM, Durcan R, Lawley S, Howe K, et al. Cardiac (123)IMIBG normal uptake values are population-specific: Results from a cohort of controls over 60 years of age. J NuCl Cardiol [Epub ahead of print] 16 Sept 2019. Available from: https://doi.org/10.1007/s12350-019-01887-6.

26 Albert MS, DeKosky ST, Dickson D, Dubois B, Feldman HH, Fox NC, et al. The diagnosis of mild cognitive impairment due to Alzheimer's disease: recommendations from the National Institute on Aging-Alzheimer's Association workgroups on diagnostic guidelines for Alzheimer's disease. Alzheimers Dement 2011; 7: 270-9.

27 McKeith I, O'Brien J, Walker Z, Tatsch K, Booij J, Darcourt J, et al. Sensitivity and specificity of dopamine transporter imaging with (123)I-FP-CIT SPECT in dementia with Lewy bodies: a phase III, multicentre study. Lancet Neurol 2007; 6: 305-13.

28 McKeith I, Ballard C, O'Brien J, Barber R, Ince P, Jaros E, et al. Predictive accuracy of clinical diagnostic criteria for dementia with lewy bodies: a prospective neuropathological validation study. Neurology 2000; 54: 1050-8.

29 Jacobson AF, Travin MI. Impact of medications on mIBG uptake, with specific attention to the heart: comprehensive review of the literature. J Nucl Cardiol 2015; 22: 980-93.

30 Benamer TS, Patterson J, Grosset DG, Booij J, de Bruin K, van Royen E, et al. Accurate differentiation of parkinsonism and essential tremor using visual assessment of [ $\left.{ }^{123} \mathrm{I}\right]-\mathrm{FP}-\mathrm{CIT}$ SPECT imaging: the $\left[{ }^{123} \mathrm{I}\right]-\mathrm{FP}-\mathrm{CIT}$ study group. Movement Disorders 2000; 15: 503-10.

31 O'Brien JT, Colloby S, Fenwick J, Williams ED, Firbank M, Burn D, et al. Dopamine transporter loss visualized with FP-CIT SPECT in the differential diagnosis of dementia with Lewy bodies. Arch Neurol 2004; 61: 919-25.

32 Lloyd JJ, Petrides G, Donaghy PC, Colloby SJ, Attems J, O'Brien JT, et al. A new visual rating scale for loflupane imaging in Lewy body disease. Neuroimage Clin 2018; 20: 823-9.

33 Massa F, Arnaldi D, De Cesari F, Girtler N, Brugnolo A, Grazzini M, et al. Neuroimaging findings and clinical trajectories of Lewy body disease in patients with MCl. Neurobiol Aging 2019; 76: 9-17.

34 Taylor JP, McKeith IG, Burn DJ, Boeve BF, Weintraub D, Bamford C, et al. New evidence on the management of Lewy body dementia. Lancet Neurol 2020; 19: 157-69.

35 Kasanuki K, Iseki E, Ota K, Kondo D, Ichimiya Y, Sato K, et al. ${ }^{123}$ I-FP-CIT SPECT findings and its clinical relevance in prodromal dementia with Lewy bodies. Eur J Nucl Med Mol Imaging 2017; 44: 358-65.

36 Thomas AJ, Attems J, Colloby SJ, O'Brien JT, McKeith I, Walker R, et al. Autopsy validation of ${ }^{123}$-FP-CIT dopaminergic neuroimaging for the diagnosis of DLB. Neurology 2017; 88: 276-83.

37 Albin RL, Fisher-Hubbard A, Shanmugasundaram K, Koeppe RA, Burke JF, Camelo-Piragua $\mathrm{S}$, et al. Post-Mortem evaluation of amyloid-dopamine terminal positron emission tomography dementia classifications. Ann Neurol 2015; 78: 824-30.

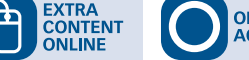
OPEN
ACCESS 